\title{
Treatment of bilateral vocal cord paralysis following permanent recurrent laryngeal nerve injury ${ }^{\text {ts }}$
}

\author{
Francesco Dispenza, MD, $\mathrm{PhD}^{\mathrm{a}, *}$, Carlo Dispenza, $\mathrm{MD}^{\mathrm{b}}$, Donatella Marchese, $\mathrm{MD}^{\mathrm{b}}$, \\ Gautham Kulamarva, MS, DNB, MRCS (Ed), DOHNS ${ }^{\mathrm{c}}$, Carmelo Saraniti, MD, $\mathrm{PhD}^{\mathrm{b}}$ \\ ${ }^{a}$ U.O. Otorinolaringoiatria-Azienda Sanitaria Provinciale Agrigento Ospedale "San Giovanni di Dio," Agrigento, Italy \\ ${ }^{\mathrm{b}}$ Dipartimento Biomedicina Sperimentale e Neuroscienze Cliniche-U.O. Otorinolaringoiatria Università degli Studi di Palermo, Italy \\ ${ }^{\mathrm{c}}$ Consultant ENT Surgeon, ENT Clinic, Nayak's Rd, Kasaragod, India
}

Received 13 May 2011

\begin{abstract}
Bilateral vocal cord paralysis is a serious illness requiring emergency intervention to resolve the potentially life-threatening respiratory distress. Several surgical procedures were proposed to help improve the airway and to eliminate the tracheostoma in those patients with permanent paralysis. All the procedures have their own advantages and disadvantages. We conducted a retrospective study of 30 patients affected by bilateral vocal cord paralysis following total thyroidectomy. All the patients underwent total thyroidectomy for benign thyroid pathology. In 26 patients $(86.6 \%)$, cord paralysis occurred during the perioperative stage; and in the remaining 4 cases $(13.3 \%)$, it occurred within the following 6 months. We treated all these bilateral recurrent laryngeal nerve paralysis patients with arytenoidectomy alone in 5 patients and arytenoidectomy with concomitant true and false posterior cordectomy in the remaining 25 patients. Twenty-four of the 25 patients who underwent the combined procedures $(96 \%)$ reported subjective respiratory improvement and were decannulated within 60 days, being able to return to their normal daily activities. This study demonstrates that arytenoidectomy associated with posterior cordectomy is a satisfactory surgical treatment of bilateral vocal cord paralysis because it leads to a considerable and stable enlargement of the breathing space.

(C) 2012 Elsevier Inc. All rights reserved.
\end{abstract}

\section{Introduction}

Bilateral vocal cord paralysis is a serious condition requiring emergency intervention to resolve the dyspnea that sometimes may become life threatening. The first option is very often a tracheotomy, performed upon admission of the patient or even in the immediate postoperative period following thyroidectomy. This latter surgical procedure is the most common cause of bilateral recurrent laryngeal nerve injury, with its consequent bilateral vocal cord paralysis [1].

Tracheotomy still remains an effective treatment, but is not acceptable as a long-term solution. Throughout the past

\footnotetext{
论 No conflicts of interest are present.

* Corresponding author. Via Oreto, 33990124 Palermo, Italy. Tel.: +39 091442084; fax: +39091442084

E-mail address: francesco.dispenza@gmail.com (F. Dispenza)
}

century, several operations were proposed to eliminate the tracheostoma in those patients with permanent paralysis. Any such surgical procedure should ideally permit recovery of the upper airway patency, with minimal impairment of other laryngeal functions. The techniques proposed to achieve this objective included both extralaryngeal and endoscopic approaches.

In 1922, the first extralaryngeal approach was proposed by Jackson [2], who described a ventriculocordectomy. Since then, Hoover [3] described a submucosal vocal cord resection; and King [4] proposed a laterofixation of the vocal cord. Another extralaryngeal technique was successively proposed by Woodman [5], who advocated arytenoidectomy associated with suture of the vocal process to the inferior cornu of thyroid cartilage by a posterolateral approach to the larynx.

However, the extralaryngeal approaches were replaced with less invasive endoscopic approaches including 
arytenoidectomy proposed by Thornell (with electrocautery) [6] and by Ossoff et al (with $\mathrm{CO}_{2}$ laser) [7] and posterior cordotomy proposed by Dennis and Kashima [8]. The arytenoidectomy was subsequently modified by Crumley [9], who described removal of the medial portion of one or both arytenoid bodies, and by Remacle et al [10], who advocated subtotal arytenoidectomy with preservation of a thin posterior portion.

A few authors found some advantage in treating this pathology by combining the 2 more commonly adopted approaches, namely, arytenoidectomy and posterior cordotomy, resulting in a more stable improvement of respiratory space [11]. Although the techniques improved progressively with time, a risk of reoperation is still present, which is often caused by granulation, tissue formation, and narrowing of airway space. Motta et al [12] in 2003 described arytenoidectomy with posterior cordectomy using $\mathrm{CO}_{2}$ laser, giving acceptable results.

In this study, we treated bilateral recurrent paralysis in adduction by arytenoidectomy and concomitant true and false posterior cordectomy; and we discuss our results.

\section{Material and methods}

We conducted a retrospective study of all the patients affected by bilateral vocal cord paralysis following total thyroidectomy. The patients underwent thyroidectomy between August 1998 and September 2009. Preoperative evaluation before the surgery for definitive management of vocal cord paralysis included clinical head and neck examination, clinical assessment of the cranial nerves, and fiberoptic laryngoscopy with assessment of laryngeal sensitivity by touching the larynx with the endoscope tip. Deglutition was assessed by videoesophagography with fluorine (in the presence of sensitivity disorders) after managing the acute dyspnea.

All patients underwent tracheostomy at the time of initial presentation with dyspnea following bilateral vocal cord paralysis. We waited for 6 to 12 months, following the cord palsy, before taking them up for definitive surgery. During this period, patients underwent scheduled periodic laryngoscopies for assessment of the possibility of spontaneous recovery.

During the initial 2 years of our study, only $\mathrm{CO}_{2}$ laser arytenoidectomy was adopted under suspension microlaryngoscopy. The procedure adopted was as follows: after incising the mucosa over arytenoids from vocal process to the apex, the arytenoid cartilage was exposed. Then the body and the vocal process were vaporized using $\mathrm{CO}_{2}$ laser. To provide even better results, the technique was changed in the subsequent years as described below: under suspension microlaryngoscopy, arytenoid cartilage was ablated using $\mathrm{CO}_{2}$ laser, paying particular attention to the interarytenoid area, along with removal of posterior third of the false cord and the true vocal cord.
In the immediate postoperative period, patients were treated with antibiotic therapy for 1 week and intravenous steroids (betamethasone $4 \mathrm{mg} / \mathrm{d}$ ) for 2 days to reduce the postoperative edema. A proton pump inhibitor therapy was initiated in all cases to reduce the risk of endolaryngeal granulomas for 6 months. Follow-up consisted of fiberoptic laryngoscopies performed on a weekly basis until complete healing of the surgical site, then every month for the first 6 months following surgery, and subsequently once a year. In addition to clinical findings regarding the glottic space, a spectrography was done to evaluate the voice, analyzing the following data: subjective evaluation, maximal phonation time, and harmonics-noise ratio. Patients were divided into 2 groups according to the surgical technique adopted: group A had unilateral arytenoidectomy, and group B had unilateral arytenoidectomy and posterior true and false cordectomy. The data regarding surgical complications, postoperative care and time of recovery, need for reoperation, and time of complete stabilization were recorded.

An informed consent was obtained from each patient before surgical procedure. Our institutional review board approved the study.

\section{Results}

There were a total of 30 patients included in the current study. The group included 22 women $(73 \%)$ and 8 men $(27 \%)$, with an average age of 53 years. In all patients, the paralysis resulted from total thyroidectomy for benign thyroid pathology. In 26 patients $(86.6 \%)$, bilateral cord paralysis occurred during the perioperative stage, whereas in 4 cases $(13.3 \%)$, a unilateral palsy was in the immediate postoperative period and the contralateral vocal cord palsy occurred within the following 6 months. The patient characteristics are summarized in Table 1.

Of the 30 patients, 28 were already having tracheostomy, whereas the remaining 2 patients underwent tracheostomy during glottis-widening surgery as a precautionary measure.

For treatment purposes, $16.7 \%$ of patients belonged to group A, whereas the remaining $83.3 \%$ were part of group B. Group A included 5 patients treated by arytenoidectomy and tracheotomy. Group B included 25 patients managed with arytenoidectomy and posterior true and false cordectomy along with tracheotomy. As regards the 5 patients undergoing laser $\mathrm{CO}_{2}$ arytenoidectomy alone (group A), only in 3 $(60 \%)$ cases of 5 was surgery fully successful, allowing for

Table 1

Patient's characteristics

\begin{tabular}{llcc}
\hline Series characteristics & No. of cases & Percentage \\
\hline Patients & Male & 8 & $26.6 \%$ \\
& Female & 22 & $73.3 \%$ \\
Etiology & Thyroid surgery & 30 & $100 \%$ \\
Paralysis onset & Perioperative period & 26 & $86.6 \%$ \\
& 15 d to 6 mo & 4 & $13.3 \%$ \\
\hline
\end{tabular}


Table 2

Tracheal cannula removal timing

\begin{tabular}{|c|c|c|c|}
\hline \multicolumn{4}{|c|}{ Tracheal cannula removal } \\
\hline Time & Intervention done & No. of cases & Total cases \\
\hline \multirow[t]{2}{*}{ Within $60 \mathrm{~d}$} & Arytenoidectomy & $3 / 5$ & 27 \\
\hline & $\begin{array}{l}\text { Arytenoidectomy }+ \text { posterior } \\
\text { cordectomy }\end{array}$ & $24 / 25$ & \\
\hline \multirow{2}{*}{$\begin{array}{l}\text { After surgical } \\
\text { revision }\end{array}$} & Arytenoidectomy & $1 / 2$ & 2 \\
\hline & $\begin{array}{l}\text { Arytenoidectomy }+ \text { posterior } \\
\text { cordectomy }\end{array}$ & $1 / 1$ & \\
\hline
\end{tabular}

decannulation within 60 days. In the remaining 2 (40\%) patients, decannulation was not possible because of a very narrow glottic space, with subsequent persistent moderate dyspnea. One of them underwent surgical revision, converting the arytenoidectomy into arytenoidectomy and posterior true and false cordectomy, and was then successfully decannulated, whereas the other patient could not undergo a surgical revision because of worsening of a preexisting respiratory failure (Table 2).

Following the partial failure of simple arytenoidectomy, all the next 25 patients (all included in group B) were treated with arytenoidectomy and posterior false and true cordectomy using $\mathrm{CO}_{2}$ laser (Fig. 1). Twenty-four patients of group B (96\%) reported subjective respiratory improvement and were decannulated within 60 days, becoming able to return to their normal daily activities (Table 2). Only one patient of group B needed a second surgery, so as to enlarge further his breathing space, thus enabling decannulation.

Quality of the voice gradually improved in all patients, following an initial worsening, thus granting them adequate

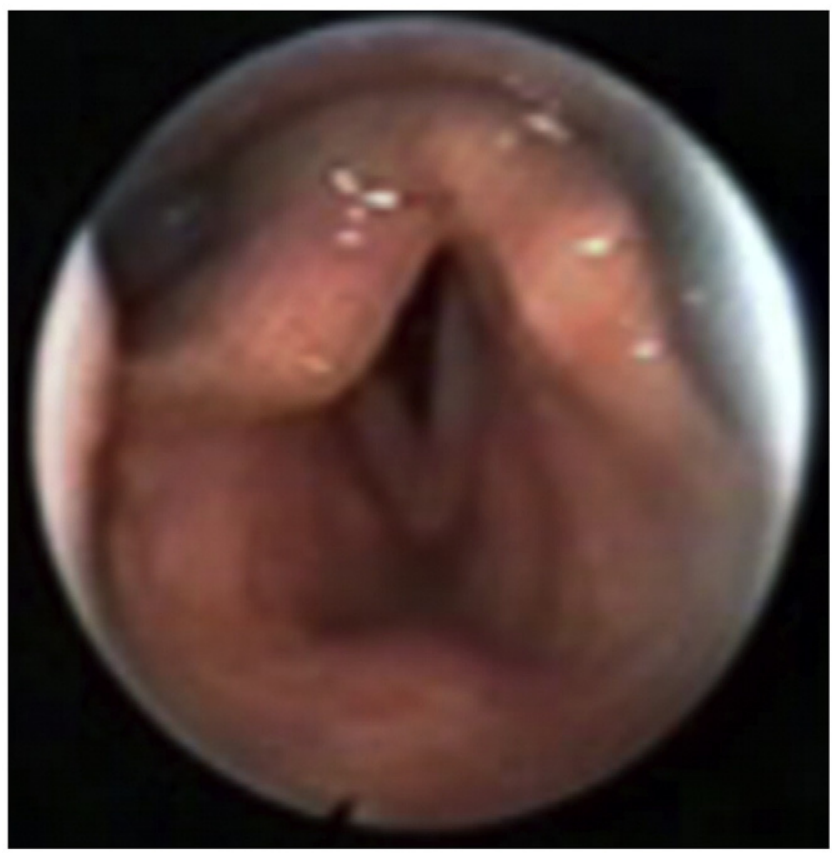

Fig. 1. Postoperative laryngoscopic view after arytenoidectomy and posterior false and true cordectomy using $\mathrm{CO}_{2}$ laser.
Table 3

Voice evaluation by spectrography

\begin{tabular}{|c|c|c|c|}
\hline & $\begin{array}{l}\text { Subjective voice } \\
\text { characteristics }\end{array}$ & $\begin{array}{l}\text { MPT } \\
(16-18 \mathrm{~s})\end{array}$ & $\begin{array}{l}\text { HNR } \\
(6-8 \mathrm{~dB})\end{array}$ \\
\hline Preoperative & $\begin{array}{l}\text { Light dysphonia } \\
\text { Normal timbre }\end{array}$ & 11 & 3.2 \\
\hline Early postoperative & $\begin{array}{l}\text { Severe dysphonia } \\
\text { Veiled timbre }\end{array}$ & 4 & -7.9 \\
\hline Stabilization & $\begin{array}{l}\text { Moderate dysphonia } \\
\text { Rough voice }\end{array}$ & 8 & -4.2 \\
\hline
\end{tabular}

Mean value of the series treated with arytenoidectomy associated with posterior cordectomy. MPT indicates maximal phonation time; HNR, harmonic-noise ratio; $\mathrm{dB}$, decibels.

communicative skills (Table 3 ). In the postoperative period, all patients, regardless of the procedure, had no dysphagia, with scarce episodes of microaspirations, resolved by deglutition rehabilitation within 1 week after surgery. There were no postoperative bleeding episodes (Table 4).

The only postoperative complication was the appearance of endolaryngeal granulations in 6 patients $(20 \%)$, which were removed under direct microlaryngoscopic procedure in 2 patients and resolved spontaneously in others.

\section{Discussion}

The main objective in treating bilateral vocal cord paralysis in adduction is obviously to enable the patient to breath normally. Nevertheless, to guarantee a decent quality of life, swallowing and phonatory functions need to be maintained, in addition to removal of the tracheostomy frequently done as an emergency act.

Spontaneous recovery of vocal cord motility is very rare as has been reported in the literature. If it happens, this is thought to be possible in cases of axonotmesis where neuroregeneration might progress toward target muscles at a speed of 1 to $3 \mathrm{~mm} / \mathrm{d}$ [13]. It has been observed by different investigators that, in patients undergoing endoscopic laryngeal surgery 6 to 12 months after the onset of paralysis, no signs of cord recovery, deriving from nerve regeneration, were noticeable in the untreated vocal cord $[14,15]$.

For those patients showing no spontaneous functional recovery, several surgical techniques to achieve a glottic space enlargement have appeared throughout the years.

Table 4

Summary of laryngeal functions results

Postoperative laryngeal functions

\begin{tabular}{lll}
\hline & Postoperative period & Stabilization \\
\hline Phonation & Deterioration & $\begin{array}{l}\text { Satisfactory for the purpose } \\
\text { of reasonable quality of life }\end{array}$ \\
$\begin{array}{l}\text { Swallowing } \\
\text { Fixation }\end{array}$ & $\begin{array}{l}\text { 1-wk dysphagia } \\
\text { Inadequate }\end{array}$ & Normal \\
\hline
\end{tabular}


Progresses have been achieved with the use of $\mathrm{CO}_{2}$ laser endoscopic surgery, leading to shorter hospitalization and higher patient compliance. Since 1984, precision microsurgery on patients with bilateral vocal cord paralysis has been possible, despite the small surgical site [7]. Among the advantages brought about by this technique are precision, microcirculatory hemostasis, and reduction of the intraoperative edema. On the other hand, disadvantages included the occurrence of possible complications, such as formation of granulomas; loss of an adequate vocal capacity, which might arise regardless of the instrument and technique used; and chondroperichondritis of the cricoid cartilage, which is specific to this surgery.

The endoscopic surgeries analyzed in this study are arytenoidectomy as described firstly by Thornell [6] and modified later by Ossoff et al [7], and arytenoidectomy with posterior true and false cord cordectomy as reported by Motta et al [12]. Combination of such techniques, in our opinion, grants the best results in terms of normal breathing with minimal deglutition impairment, also allowing for an acceptable quality of voice. Removal of arytenoid is associated with an almost unchanged voice quality, but the space gained is often insufficient. As reported in the literature, less invasive surgeries, despite preserving the phonatory function, tend to impair breathing and require several surgical revisions [9].

Posterior third of the vocal cord is another surgical site of interest: the posterior cordotomy technique of Dennis and Kashima [8] consists of creation of a wedge-shaped opening in both the true and false vocal cord by retracting the thyroarytenoid muscle. It is a simple surgery, which leads to a predictable enlargement of the glottic space while reducing to a minimum the trauma for the structures involved and preserving swallowing functions. The arytenoidectomy and posterior cordectomy of Motta et al, with removal of the posterior third of both the true and false vocal cord, provide the best results but entail higher phonatory impairment. However, the voice quality is of secondary importance in patients with persisting dyspnea that worsens under physical effort. Even then, the voice quality, although worsened, is adequate to grant a relatively good quality of life. Removal of the posterior third of true and false cord, instead of a simple cordotomy, reduces the incidence of restenosis of the glottic space. In fact, the main problem that leads patients to a reoperation is not a voice problem or dysphagia, but the narrowing of the laryngeal space obtained after one of the abovedescribed procedures.

As regards this study, 22 of the 30 patients were women, reflecting probably the incidence of benign thyroid pathologies in the general population. Forty percent of the patients undergoing simple arytenoidectomy required surgical revision to enlarge the glottic space, irrespective of voice result. However, those patients undergoing both arytenoidectomy as well as posterior true and false cordectomy had only a $4 \%$ failure rate. The results concerning the latter group of patients were undoubtedly better. All patients returned to their daily activities, and only one needed surgical revision. Obviously, in a pathology like this, it is difficult to achieve the numbers required to achieve any statistical significance.

\section{Conclusions}

A disabling condition like bilateral vocal cord palsy needs an intervention that can improve the airway as well as maintain reasonable phonatory function. This study demonstrates that arytenoidectomy associated with posterior cordectomy is a satisfactory surgical treatment of this condition, leading to a considerable and stable enlargement of the breathing space and acceptable voice quality with minimal aspiration, if any, whereas arytenoidectomy alone tends to be insufficient in alleviating the pathology as well as having an increased rate of revision.

\section{References}

[1] Yumoto E, Minoda R, Hyodo M, et al. Causes of recurrent laryngeal nerve paralysis. Auris Nasus Larynx 2002;29:41-5.

[2] Jackson C. Ventriculocordectomy, a new operation for the cure of goitrous glottic stenosis. Arch Surg 1922;4:257-74.

[3] Hoover WB. Bilateral abductor paralysis, operative treatment of submucus resection of the vocal cord. Arch Otolaryngol 1932;15: 337-55.

[4] King BT. A new and function restoring operation for bilateral abductor cord paralysis. JAMA 1939;112:814-23.

[5] Woodman D. A modification of the extralaryngeal approach to arytenoidectomy in bilateral abductor vocal cord paralysis. Arch Otolaryngol 1946;43:63-5.

[6] Thornell WC. Intralaryngeal approach for arytenoidectomy in bilateral abductor vocal cord paralysis. Arch Otolaryngol Head Neck Surg 1948;47:505-8.

[7] Ossoff RH, Sisson GA, Duncavage JA, et al. Endoscopic laser arytenoidectomy for the treatment of bilateral vocal cord paralysis. Laryngoscope 1984;94:1293-7.

[8] Dennis DP, Kashima HK. Carbon dioxide laser posterior cordectomy for treatment of bilateral vocal cord paralysis. Ann Otol Rhinol Laryngol 1989;98:930-4.

[9] Crumley RL. Endoscopic laser medial arytenoidectomy for airway management in bilateral laryngeal paralysis. Otolaryngol Clin North Am 1993;102:81-4.

[10] Remacle M, Lawson G, Mayne A, et al. Subtotal carbon dioxide laser arytenoidectomy by endoscopic approach for treatment of bilateral cord immobility in adduction. Ann Otol Rhinol Laryngol 1996;105:438-45.

[11] Bizakis JG, Papadakis CE, Karatzanis AD, et al. The combined endoscopic $\mathrm{CO}_{2}$ laser posterior cordectomy and total arytenoidectomy for treatment of bilateral vocal cord paralysis. Clin Otolaryngol 2004;29:51-4.

[12] Motta S, Moscillo L, Imperiali M, et al. $\mathrm{CO}_{2}$ laser treatment of bilateral vocal cord paralysis in adduction. ORL 2003;65:359-65.

[13] Seddon HJ. Three types of nerve injuries. Brain 1943;66:237.

[14] Maurizi M, Paludetti G, Galli J, et al. $\mathrm{CO}_{2}$ laser subtotal arytenoidectomy and posterior true and false cordotomy in the treatment of post-thyroidectomy bilateral laryngeal fixation in adduction. Eur Arch Otorhinolaryngol 1999;256:291-5.

[15] Rovò L, Jori J, Brzozka M, et al. Airway complication after thyroid surgery: minimally invasive management of bilateral recurrent nerve injury. Laryngoscope 2000;110:140-4. 